\section{Alcohol overuse: cutting to the bone}

Building on several decades of research, Dr Martin Ronis,
a Professor at the Louisiana State University Health Sciences Center-New Orleans explores the effects of alcohol on bone turnover His current research mins to better un forthe toxic effects of alcohol, particularly in women, with a view to uncovering vital therapeutic targets to prevent bone loss. As alcohol-induced bone loss shares many features in common with bone loss during menopause and ageing, his work provides fundamental insights into the regultion of bone growth and turnover.

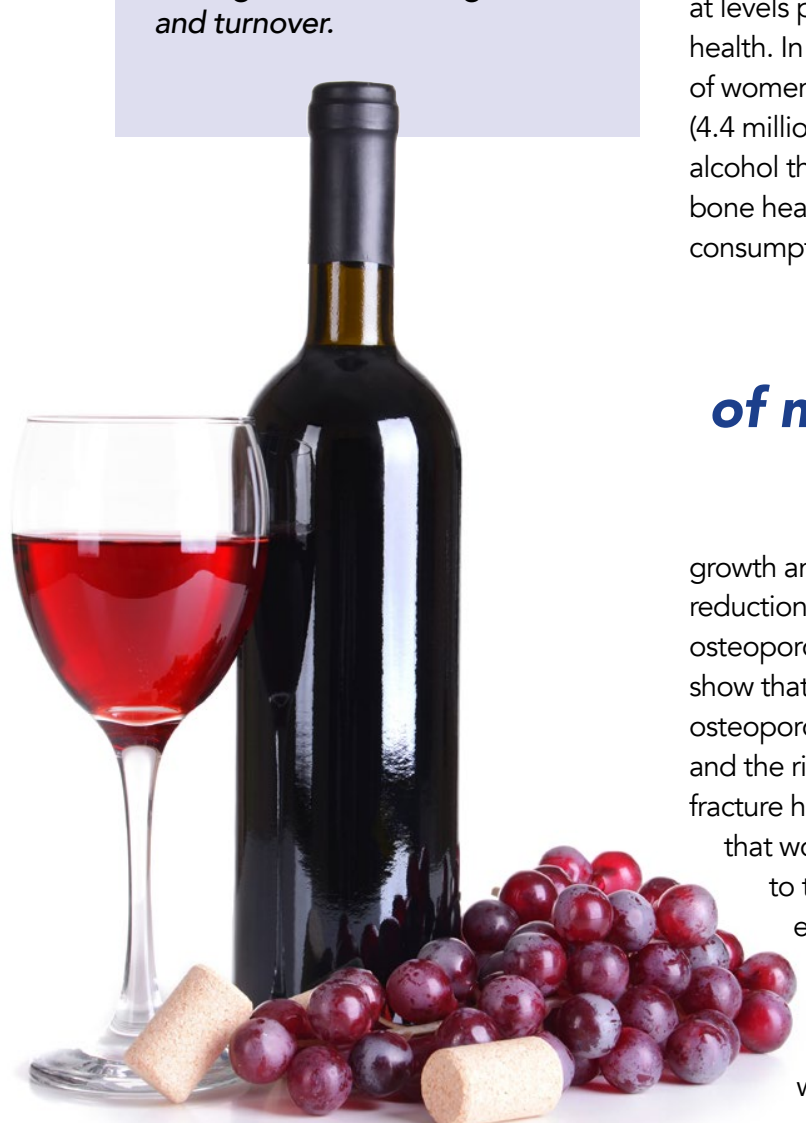

ur bones are living tissues that replacement (bone remodelling) ven into adulthood. As we grow in childhood, our bone mass increases until it reaches 'peak bone mass' (PBM). Defined as the greatest amount of bone in tote teens and ealy 20s. Individuls with a hins and 20 . Ind osteoporosis a bone disease that occurs when the body loses too much bone, makes too little bone, or both) a leading cause of morbidity and mortality in older post-menopausal women.

ALCOHOL AND BONE HEALTH Although the majority of people drink within safe limits, many consume alcoho at levels proven to be harmful to their . 18-30 years million) binge drink. Drinking more (A.4 (interere with bone

\section{BETTER BONES}

This is where the research of DrMartin Ronis, Professor in the Department of Pharmacology and Experimenta Therapeutics at the Louisiana State New Orsity Health Sciences CenterNew Orleans comes in. His research explores the effects of alcohol on bone in the relationship between inhibition of bone formation and stimulated bone resorption associated with alcohol abuse in young women and the increased risk of osteoporosis - resulting from a failure to attain PBM. His research involves multidisciplinary basic sciences, including whole animal physiology, nutrition and endocrinology, pathology and molecular biological techniques. Ultimately, Prof Ronis aims to better understand the molecular basis for the toxic actions of alcohol, which might uncover new therapeutic targets to prevent bone loss Prof Ronis' current work builds on long history of alcohol research with

\section{Osteoporosis is a leading cause} of morbidity and mortality in older postmenopausal women.

collaborators at the Department of

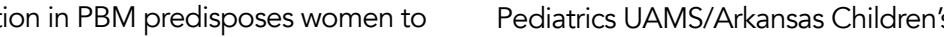
Hospital. The researchers explored oporosis, a decreased bone mass for the risk of fractures, and can delay ture healing. Although it is well know women are more susceptible the toxic effects of alcohol (i.e. thanol) than men, much less is known about the mechanisms of alcohol overuse in women with respect to their bone health. They found that bone density, cortical area, and mature bone strength were negatively affected by alcohol, and showed for the first time that formation during limb lengthening They also dung limb lengthening. They also demonstrated that certain cytokines - play an important role in the process of ethanol-related skeletal pathology. Excitingly, the team were able to protect fracture healing using inhibitory ntibodies against these cytokines

in the same rat models.

This early work combined with the fact that alcoholism is a known risk factor for osteoporosis led Prof Ronis to think about ways in which reduced bone mass (osteopenia) occurs after chronic alcoho consumption, leading to increased risk of osteoporosis in ageing. Since osteoporosis is predominantly a disease that aflects women, Prof Ronis decided to focus his research efforts on females including cycling pregnancy and potctation) dovetaling with anotherof his research into alcoholnutritional interactions in pregnancy, funded by the National Institute on Alcohol Abuse and Alcoholism.

ETHANOL EFFECTS IN PREGNANCY a study published in 2006, Prof Ronis directly tested the effect of ethanol in pregnancy and cycling rats. The team measured the effect of alcohol on skeletal parameters including tibial bone mineral density (TBMD), bone mineral content and bone mineral area (BMA). A dose-dependent skeletal toxicily following alcohol exposure was observed,

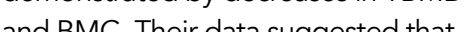
and BMC. Their data suggested that rats is mainly due to inhibited bone formation In striking contrast, bone loss in non-pregnant rats was higher than in that observed in pregnant rats and occurred through a different mechanism concomitant with additional increases in bone resorption and a decrease in circulating estradiol levels.

PROTECTIVE ESTRADIOL LEVELS effect of alcohol further, Prof Ronis and his team demonstrated the role of osteoclastogenesis - the process whereby osteoclast cells are develope for breaking alla cells responsible for breaking down bone, resulting in bone rorption. Prof Ronis showed that
ETHANOL EFFECTS ON BONE TURNOVER

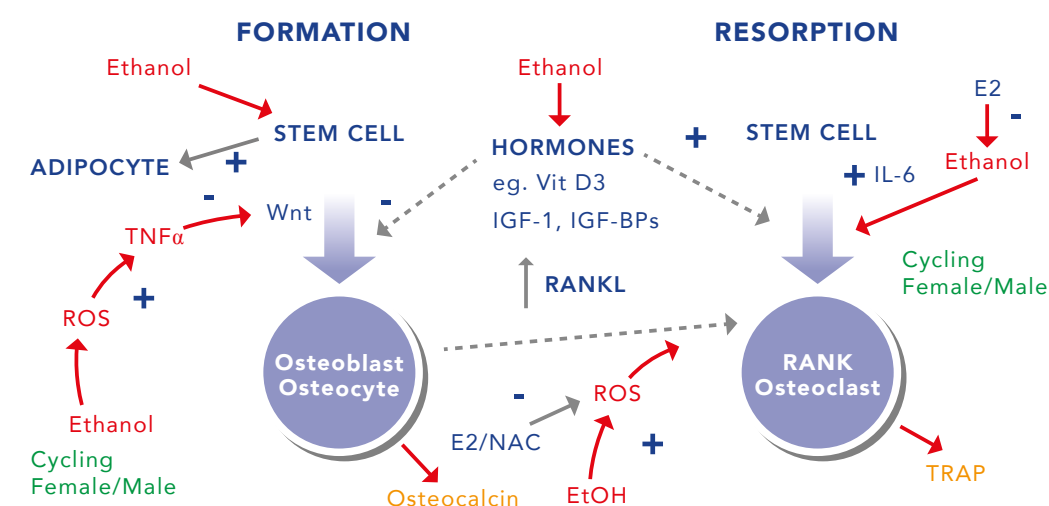

Preanancy
Post-Weaning REDUCED NEW

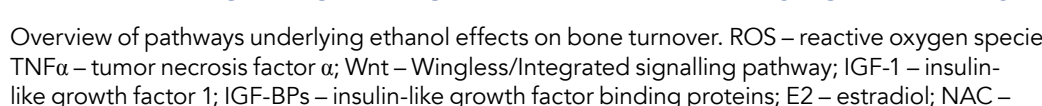

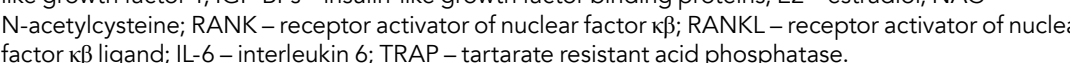

Prof Ronis' current work aims to unpick in fine detail, the ROS signalling mechanisms in bone after excess ethanol consumption.

oxidative stress, which in turn promotes that they may be at increased risk RANKL (receptor activator of nuclear factor $k \beta$ ligand, a substance neede for osteoclast formation) - inducing osteoclastogenesis. This finding has significant implications for the use of contraceptive pills containing alcoholic osteopenia (weag bons) in individuals who drink excessively. Notably the team also showed that anabolic by alcohol in post-lactation female rats. This has important implications for postlactating women who resume drinking after stopping breast feeding, suggesting hat they may be at increased
of osteoporosis with age. THE ROLE OF OXIDATIVE STRESS Intrigued by the differences in bone remodelling during specific physiolog states (pregnancy, lactation and to explore the underlying molecular mechanisms further. In an elegant series of experiments, the team showed via a shift in the differentiation of bone marrow mesenchymal stem cells. Rather than forming osteoblasts (bone cell forming cells), these stem cells instead

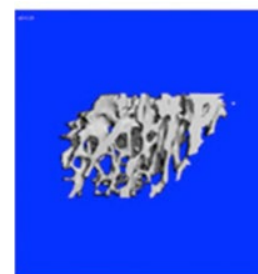

WT Pair Fed



WT EtOH

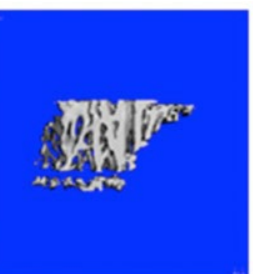

Nox4 Pair Fed

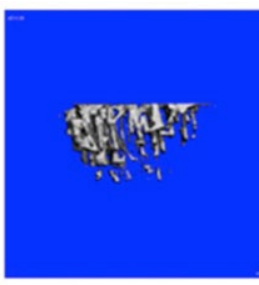

Nox4 EtOH

\section{Micro-computerized tomography images of mouse tibial trabecular bone demonstrating loss of bone
volume after ethanol (EtOH) consumption is greater in}


differentiate into fat cells or adipocytes. They an important mech oxidatives The team then went on to show that the production of excess reactive oxygen species (ROS) production by NADPH oxidase (NOX) enzymes was responsibb for the effects of ethanol on bone turnover. Experiments of bone loss in female rats and mice fed ethanol was blocked by dietary antioxidants including $\mathrm{N}$-acetylcysteine (NAC), vitamin $\mathrm{E}$ and also DPI an inhibitor of NADPH oxidase (NOX) enzymes.

Interestingly, Prof Ronis found that the effects of ethanol to inhibit osteoblastogenesis the production and stimulate bone marrow adipose cell formation were not blocked in

experimental genetic mice that lacked a active form of NOX1/2 enzymes. Since a major component of ethanol pathology in bone is the generation of excess ROS, this led the team to hypothesise that another source of ROS mediates this effect. The researchers subsequently explored the contribution of ROS-generating NADPH oxidase-4 (NOX4) and mitochondrialderived ROS in driving ethanol-induced suppression of bone formation.

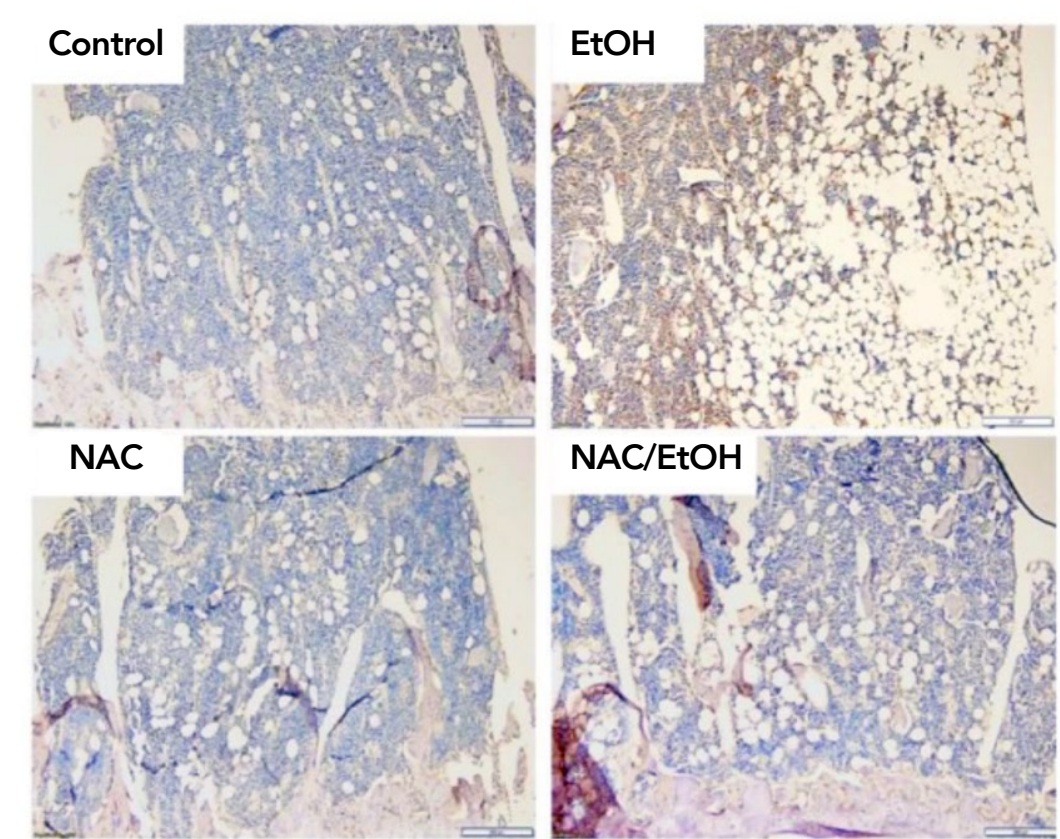

Immunohistochemical staining for nitrotyrosine protein adducts (brown) in female mouse tibial bone
following consumption of ethananol (EtOH) with or without co-treatment with the dietary antioxidant

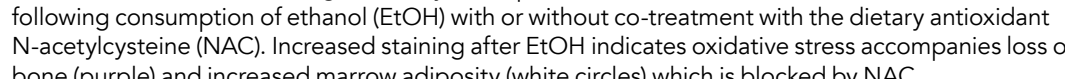

Control + NAC antioxidant $\mathrm{N}$-acetylcysteine (NAC)

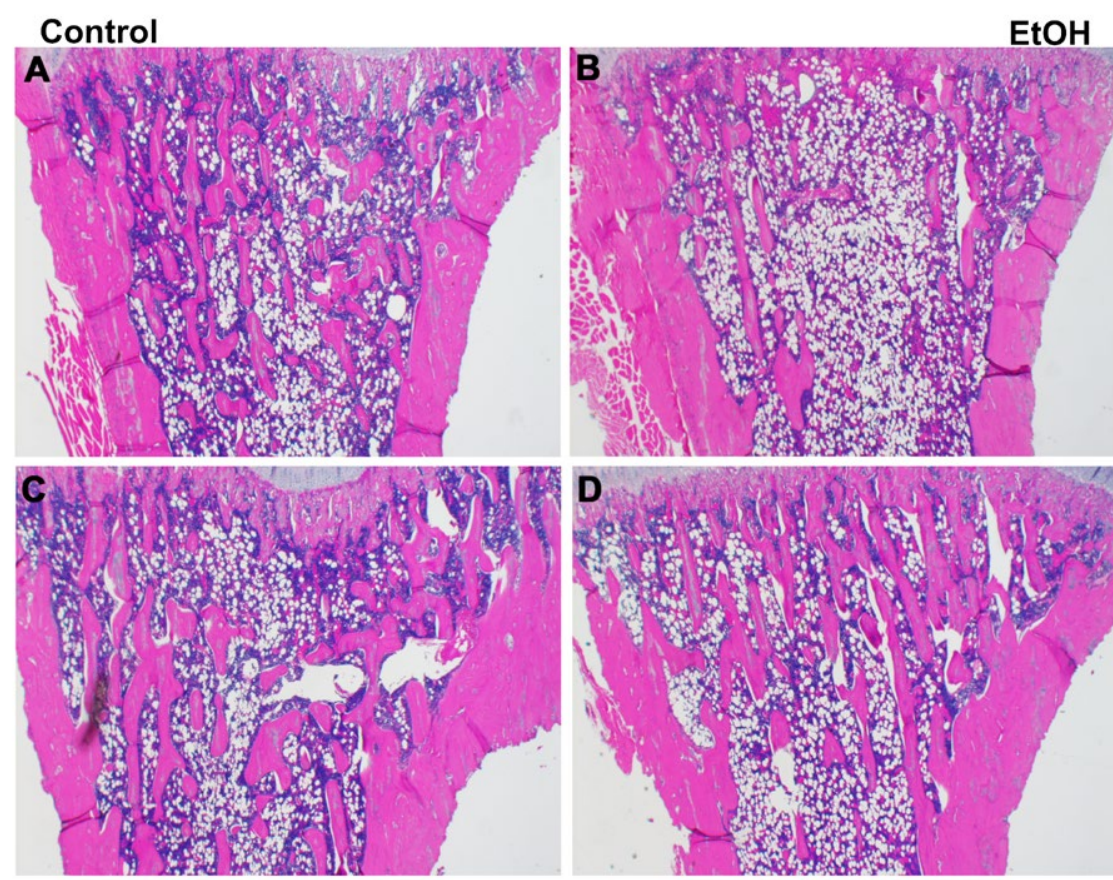

$\mathrm{EtOH}+\mathrm{NAC}$

H2E stained images of decalcified tibial bone from post-lactating female rats at the growth plate.
Pink staining - mineralised bone, outside - cortical bone, inside - columns of trabecular bone. White circles - adipocytes (fat cells in the bone marrow. Loss of bone after ethanol (EtOH) consumption is
accompanied by increased marrow adiposity (B) and this is reversed by co-treatment with the dietary

\section{URRENT RESEARCH} genetic mice that lack NOX 4 in from bone cell types (lockeoblas 4 in selected osteocytes, osteoclasts) will drill down on the mechanisms involved.

The teams' recent findings suggest that dietary antioxidants prevent alcoholinduced osteopenia as a result of blocking excess hydrogen peroxide. produced as the result of the activation of NADPH oxidase enzymes following the metabolism (breakdown) of ethanol. Importantly, since alcohol-induced bone loss shares many features in common with bone loss during menopause and ageing molecular studies of alcohol actions on hydrogen peroxide downstream effects in bone may well provide fundamental insights into common molecular pathways underlying the regul
growth and turnover. Dr Martin Ronis

References

Brown EC., Perrien DS., et al. (2002). 'Skeletal Toxicity Associated With Chronic Ethanol Exposure in a Rat Model Using
Nutrition'. J Pharm and Exp Therapy, 301;3 1132-8.

Perrien, DS., Wahl, EC., et al. (2004). 'IL-1 and TNF Antagonists Prevent Inhibition of Fracture Healing
Toxicological Sciences, 82, 656-660.

Shankar K, et al. (2006). 'Physiologic and genomic analyses of nutrition-ethanol interactions during gestation: implications for fetal ethanol toxicity. Exp Biol Med, 231:1379-1397. Shankar K, et al. (2006). 'Different molecular mechanisms underlie ethanol-induced bone loss i cycling and pregnant rats'. Shankar K, Ronis, MJJ, Badger TM. (2007). 'Effects of Pregnancy 1:55-59.

Chen J-R, et al. (2008). 'Protective Effects of Estradiol on EthanolInduced Bone Loss Involve Inhibition of Reactive Oxygen Species Extracelliluar Signal-Regusts and Downstream Activation of the Extracellular Signal-Regulated Kinase/Signal Transducer and KB Ligand Signaling Cascade.' J Pharmacol. Exp Therap 324, No 1:50-9.

Chen, J-R, et al. (2010) 'A role for ethanol-induced oxidative stress in controlling lineage commitment of mesenchymal stromal cells through inhibition of Wntbeta catenin signaling'. J. Bone Min Res, 25. $1117-1127$.

Mercer KE, et al. (2014). 'Loss of functional NADPH oxidase 2 protects against alcohol-induced bone resorption in female
p47phox-l-mice chronically fed ethanol'. Alc. Clin. Exp. Res., 38 : $672-682$.

Chen J-R, et al. (2015). 'Deficient Nox2-dependent ROS signaling acceler,

Alund, A, et al. (2016). 'Reactive oxygen species differentially regulate bone turnover in an age-specific manner in catalase transgenic female mice' J. Phom

Alund, A.W., Mercer, K.E., Pulliam, C.F., Suva, L.J., Chen, J-R., antioxidants against ethanol-induced osteopenia and chang bone morphology in female mice'. Alc. Clin. Exp. Res, 41: 46-56. Watt, J., Alund, A.W. Pulliam, C.F., Mercer, K.E., Suva, L.S., Chen,
$J$-R. and Ronis M.J.J. (2018). 'NOX deletion in mice exacerbates the effect of ethanol on trabecular bone loss and osteoblast colony formation' J. Pharmacol. Exp. Ther 366: 46-57.

¿ LsuHealthNewOrleans

/ronis_home.aspx

\section{Research Objectives}

The goal of Dr Ronis' research is to better understand the molecular basis for the toxic actions of alcohol, particularly particular, the results from research aim to link the deleterious effects of alcohol consumption on bone in women of reproductive age with increased risk of osteoporosis, a major
cause of morbidity and mortality in post-menopausal women.

\section{Detail}

Dr Martin Ronis, LSU Health Sciences Center - New Orleans, Derdido Str. New Orleans LA 70112, USA.

Martin Ronis B.A., M.A. Nat. Sci. Cantab., PhD is a tenured full Professor in the Department of Pharmacology \& Experimental Therapeutics at the Louisiana State University Wenth Sciences Center - New Orleans. He is a current NIAAA) MERIT awardee.

Funding

AIH(NIAAA): R01 AA012928, R01 AA012819, R01

Ronis Lab:

Drs Mats Hidestrand, Kartik Shankar, Jin-Ran Chen, Kelly Sciences and Drs James Watt, Kim Pedersen, Louisiana Collaborators:

Collaborators: the University of Arkansas for Medical Sciences and Dr Larry Suva, Texas A\&M University.

\section{Personal Response}

Your contribution to the field of alcohol toxicology is immense. What's next for your research?

II In addition to my current work on alcohol and on alcoholic and non-alcoholic fatty liver disease. Recently, we have focused on the role of nutrition in the progression of liver pathology to liver cancer. Interestingly, we find that both alcohol and saturated

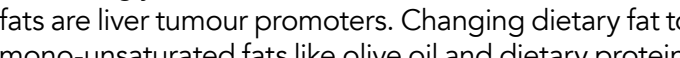
from dairy to soy protein can protect Protection appears linked to changes in gut microbiota. We are currently studying these links between diet, gut bacteria and liver cancer. In additition, we are expanding our studies on skeletal toxicity beyond alcohol to look polychlorinated biphenyls. 